\title{
(2) OPEN ACCESS \\ Comparison of retinal vessel diameter measurements from swept-source OCT angiography and adaptive optics ophthalmoscope
}

\author{
Xinwen Yao @ $1,2,3$ Mengyuan $\mathrm{Ke}^{3}{ }^{3}$ Yijie Ho, ${ }^{4}$ Emily Lin, ${ }^{3}$ Damon W K Wong, ${ }^{1,2,3}$ \\ Bingyao Tan, ${ }^{1,2,3}$ Leopold Schmetterer (1) , 2,3,5,6,7,8,9 Jacqueline Chua (1) $3,8^{3}$
}

${ }^{1}$ Institute of Health

Technologies, Nanyang Technological University, Singapore

${ }^{2}$ SERI-NTU Advanced Ocular Engineering (STANCE), Singapore

${ }^{3}$ Singapore Eye Research Institute, Singapore National Eye Center, Singapore

${ }^{4}$ Yong Loo Lin School of Medicine, National University of Singapore, Singapore ${ }^{5}$ School of Chemical and Biomedical Engineering, Nanyang Technological University, Singapore

${ }^{6}$ Department of Clinical Pharmacology, Medical University of Vienna, Vienna, Austria

${ }^{7}$ Center for Medical Physics and Biomedical Engineering, Medica University of Vienna, Vienna Austria

${ }^{8}$ Academic Clinical Program, Duke-NUS Medical School, Singapore

${ }^{9}$ Institute of Ophthalmology, Basel, Switzerland

Correspondence to Dr Jacqueline Chua, Singapore Eye Research Institute, Singapore National Eye Centre, 169856, Singapore; jacqueline.chua.y.m@seri. com.sg

Received 18 February 2020 Revised 14 April 2020 Accepted 1 May 2020 Published Online First 27 May 2020

\section{ABSTRACT \\ Background/ims To compare the retinal vessel} diameter measurements obtained from the swept-source optical coherence tomography angiography (OCTA; Plex Elite 9000, Carl Zeiss Meditec, USA) and adaptive optics ophthalmoscope (A00; RTX1, Imagine Eyes, France). Methods Fifteen healthy subjects, $67 \%$ women, mean age (SD) 30.87 (6.19) years, were imaged using OCTA and $\mathrm{AOO}$ by a single experienced operator on the same day. Each eye was scanned using two OCTA protocols $\left(3 \times 3 \mathrm{~mm}^{2}\right.$ and $\left.9 \times 9 \mathrm{~mm}^{2}\right)$ and two to five AOO scans $\left(1.2 \times 1.2 \mathrm{~mm}^{2}\right)$. The OCTA and AOO scans were scaled to the same pixel resolution. Two independent graders measured the vessel diameter at the same location on the region-of-interest in the three coregistered scans. Differences in vessel diameter measurements between the scans were assessed.

Results The inter-rater agreement was excellent for vessel diameter measurement in both OCTA protocols (ICC=0.92) and AOO (ICC=0.98). The measured vessel diameter was widest from the OCTA $3 \times 3 \mathrm{~mm}^{2}(55.2 \pm 16.3 \mu \mathrm{m})$, followed by OCTA $9 \times 9 \mathrm{~mm}^{2}(54.7 \pm 14.3 \mu \mathrm{m})$ and narrowest by the AOO $(50.5 \pm 15.6 \mu \mathrm{m} ; \mathrm{p}<0.001)$. Measurements obtained from both OCTA protocols were significantly wider than the AOO scan (OCTA $3 \times 3 \mathrm{~mm}^{2}$ : mean difference $\Delta=4.7 \mu \mathrm{m}$, $\mathrm{p}<0.001$; OCTA $\left.9 \times 9 \mathrm{~mm}^{2}: \Delta=4.2 \mu \mathrm{m}, \mathrm{p}<0.001\right)$. For vessels $>45 \mu \mathrm{m}$, it appeared to be larger in OCTA $3 \times 3 \mathrm{~mm}^{2}$ scan than the $9 \times 9 \mathrm{~mm}^{2}$ scan $(\Delta=1.9 \mu \mathrm{m}$; $p=0.005)$, while vessels $<45 \mu \mathrm{m}$ appeared smaller in OCTA $3 \times 3 \mathrm{~mm}^{2} \operatorname{scan}(\Delta=-1.3 \mu \mathrm{m} ; \mathrm{p}=0.009)$

Conclusions The diameter of retinal vessels measured from OCTA scans were generally wider than that obtained from AOO scans. Different OCTA scan protocols may affect the vessel diameter measurements. This needs to be considered when OCTA measures such as vessel density are calculated.

\section{INTRODUCTION}

Optical coherence tomography angiography (OCTA), a functional extension of OCT, offers a three-dimensional high-resolution visualisation of the retinal vasculature networks via motion contrast from the blood cells. ${ }^{1}$ Since its approval by FDA in the late 2016, the OCTA has gained immense interest among clinicians for its ability to highlight vascular alterations in retinal conditions..$^{2-4}$ Despite its potential as a microvasculature imaging tool, it remains unknown whether a retinal vessel imaged by OCTA reflects its actual diameter. Retinal vessel calibre measurements may provide pertinent information on the health status of the eye and the body. ${ }^{5} 6$ Specifically, vessel calibre measurements have been correlated with worsening of eye diseases such as diabetic retinopathy ${ }^{7}$ and systemic health status in hypertension, ${ }^{8}$ diabetic nephropathy ${ }^{9}$ and cardiovascular disease. ${ }^{10}$ Therefore, accurate and reliable measurements of the retinal vessel calibre with OCTA may be attractive. Recently, Ghasemi Falavarjani et $a l^{11}$ compared the vessel diameter measurements obtained from OCTA scans and fundus photographs, and reported that the vessels measured from OCTA scans appeared to be nearly $20 \mu \mathrm{m}$ thicker when compared with fundus photographs. However, absolute vessel diameter measurement obtained from fundus photography can be influenced by various factors such as illumination wavelength and vessel orientation. ${ }^{12} 13$ In addition, the impact of different OCTA scan protocols on the vessel diameter measurement remains unknown.

Adaptive optics ophthalmoscope (AOO) is a fundus camera system inbuilt with an adaptive optics (AO) to compensate optical aberration induced by the anterior segment of the eye. ${ }^{14}$ The AO consists of a deformable mirror in the illumination pathway and a wavefront sensor in the detection pathway, working in a feedback loop that corrects optical aberration in real time. Compared with the fundus camera, AOO allows for retinal vessel imaging with higher transverse resolution. It offers distinct delineation of the vessel wall and lumen, ${ }^{15}$ and may be less susceptible to segmentation error, ultimately allowing for more accurate vessel diameter measurements.

In our study, we compare the vessel diameter measurements using two different OCTA scan protocols with the high-resolution $\mathrm{AOO}$ as a reference and investigate if OCTA scan protocols may affect vessel diameter measurement.

\section{METHODS \\ Study participants}

A total of 15 healthy volunteers, aged 21 and above, with no history of systemic or ocular diseases, were recruited at the Singapore Eye Research Institute between June 2019 and August 2019. All individuals were screened for suitability before recruitment. Written informed consent was obtained from all participants and all procedures performed were in accordance with the ethical standards of the 
SingHealth Centralised Institutional Review Board and in accordance with the Declaration of Helsinki.

\section{Ocular examination}

Detailed interviewer-administered questionnaire was used to screen for any chronic medical history (eg, diabetes, hypertension, high cholesterol) and ocular history (eg, glaucoma, retinopathies or any surgery or laser treatment). ${ }^{16}$ Participants were then assessed for their refractive error using an axial (Canon RK-5 Autorefractor Keratometer; Canon Inc., Tokyo, Japan), and intraocular pressure. ${ }^{16}$ Their pupils were dilated with $1 \%$ tropicamide before undergoing the relevant imaging-related tests, including fundus photography, OCT and swept-source OCTA (SS-OCTA). Fundus photographs further documented the absence of any ocular diseases. ${ }^{17} 18$

\section{Optical coherence tomography angiography imaging}

OCTA imaging was performed with a prototype SS-OCT instrument (Plex Elite 9000, Carl Zeiss Meditec, USA), which employs a wavelength swept source and detects the interference signal by a photodetector. ${ }^{4}$ The light source has a central wavelength at $1050 \mathrm{~nm}$ with a scanning rate of $100000 \mathrm{~A}$-scans per second. The axial and lateral resolutions in tissue are $6.3 \mu \mathrm{m}$ and $20 \mu \mathrm{m}$, respectively. The system is integrated with a line-scan ophthalmoscope eye tracker to compensate the artefacts caused by blinking, bulk motion and involuntary eye motion. All eyes were scanned using two OCTA scan protocols: three $3 \times 3 \mathrm{~mm}^{2}$ scans with the scan density of $300 \times 300$ pixels and a uniform pitch of $10 \mu \mathrm{m} / \mathrm{pixel}$, and one $9 \times 9 \mathrm{~mm}^{2}$ scan with the scan density of $500 \times 500$ pixels and a uniform pitch of $18 \mu \mathrm{m} /$ pixel. Both OCTA protocols used four repeated $\mathrm{B}$-scans to generate en face OCTA images. Among the three $3 \times 3 \mathrm{~mm}^{2}$ scans, one was centred on the macular area, with the other two superior and inferior to the macular region, respectively. The single $9 \times 9 \mathrm{~mm}^{2}$ scan was centred on the macular. The OCTA scans used in this study were generated from the superficial vascular plexus from the inner limiting membrane to the inner plexiform layer by the PLEX Elite Review Software V.1.7.1.31492.

\section{Adaptive optics ophthalmoscope imaging}

The AOO (RTX1, Imagine Eyes, France) is essentially an AO fundus camera system that uses wide field (flood) illumination and a two-dimensional camera as detector. ${ }^{14}$ It employs an AO unit comprised of a deformable mirror and a Shack-Hartmann wavefront sensor. The shape of the deformable mirror is controlled by the feedback from the wavefront sensor, to correct the optical aberration and increase the resolution as well as light throughput. The main advantage of AOO over existing AO scanning laser ophthalmoscopes (AOSLOs) is its higher imaging speed that empowers a stronger resistance to motion artefacts. During acquisition, the AOO captures 40 high-resolution twodimensional en face image and stabilises the frames using automatic image registration. The total acquisition time is about $2 \mathrm{~s}$. It then generates an averaged image using the stabilised frames. The system has a lateral resolution of $2 \mu \mathrm{m}$ on the retina, and a field of view of $4^{\circ} \times 4^{\circ}$, equivalent to $1.2 \times 1.2 \mathrm{~mm}^{2}$. The digitised image has a uniform pitch of $0.8 \mu \mathrm{m}$. The accessible area of AOO is about $9 \times 6 \mathrm{~mm}^{2}$ on the retina centred at the fovea. For each eye, three to five AOO scans were focused on arterioles/venules, with the selection of the region of interest (ROI) guided by the OCTA $9 \times 9 \mathrm{~mm}^{2}$ scan.

\section{Image processing and vessel calibre measurement}

Figure 1 shows the manually registered scans from the two OCTA protocols and one AOO scan of the same eye, and the vessel diameter measurements made from the three coregistered scans. The AO and OCTA scans were directly exported from the instruments, respectively, and analysed in ImageJ (National Institutes of Health, Bethesda, Maryland, USA). The $9 \times 9 \mathrm{~mm}^{2}$ (figure $1 \mathrm{~A}$ ) and $3 \times 3 \mathrm{~mm}^{2}$ (figure $1 \mathrm{~B}$ ) OCTA scans were rescaled to match the pitch size of the AO scan (figure 1C) using bilinear interpolation. Both OCTA scans were then manually registered

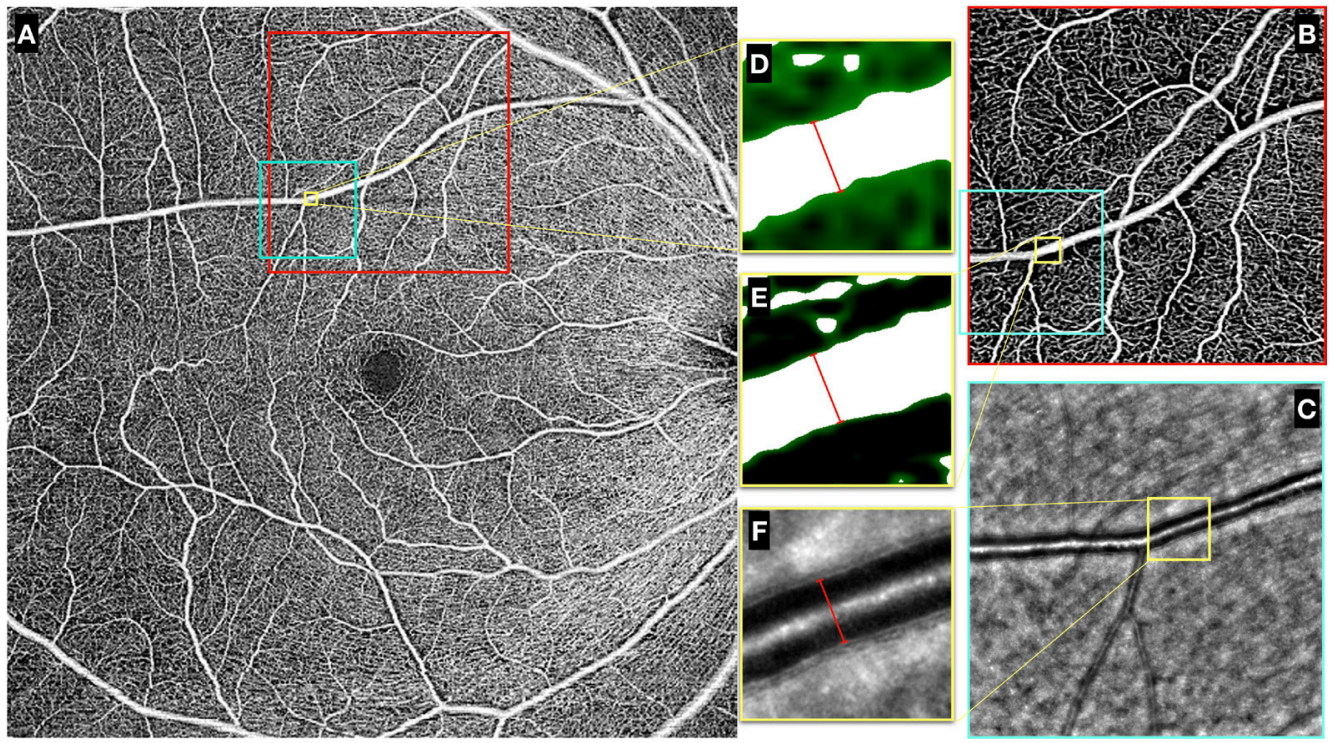

Figure 1 Example of the manually registered OCTA $9 \times 9 \mathrm{~mm}^{2}, 3 \times 3 \mathrm{~mm}^{2}$ and AOO scans on the same eye. (A) The OCTA $9 \times 9 \mathrm{~mm}{ }^{2}$ scan centred at the macular area, marked with the red box representing the corresponding $3 \times 3 \mathrm{~mm}^{2}$ scan displayed in (B) and the light blue box representing the corresponding $\mathrm{AOO}$ scan displayed in (C). (D-F) The ROls for performing vessel diameter measurement indicated by the yellow boxes taken from the (D) OCTA $9 \times 9 \mathrm{~mm}^{2}$, (E) $3 \times 3 \mathrm{~mm}^{2}$ and (F) AOO scans. For the OCTA scans, binarisation masks (green) were imposed on the original images in (D) and (E). Vessel diameter measurements were made perpendicular to the vessel direction, as shown by the red lines. A00, adaptive optics ophthalmoscope; OCTA, optical coherence tomography angiography; ROIs, regions of interest. 
to the AO scan, and binarised by using Otsu's global thresholding method. ${ }^{19}$ For every registered scan set, one ROI $(260 \times 260$ pixels) was selected on each vessel that was well captured by both instruments (figure 1A-C yellow boxes). Two independent graders $(\mathrm{MK}, \mathrm{YH})$ randomly selected one specific location along the vessel within the ROIs of three registered scans and measured the vessel diameter in the ROIs, respectively (figure 1D-F). For the OCTA scans, the vessel borders were determined based on the binarised images, and the vessel diameter was measured perpendicular to the borders (figure 1D). For AOO scans, the borders of the vessel lumen were determined based on the local image contrast, and the vessel lumen diameter was measured to represent the vessel diameter (figure 1F). Each grader made three random measurements along the vessel in the ROI and the averaged vessel diameter was reported for analysis.

\section{Statistical analysis}

The intraclass correlation coefficients (ICCs) and 95\% CIs were used to assess the absolute agreement (1) between raters and (2) between the vessel diameter measurements from different types of scans. ICC values $<0.5$, between 0.5 and 0.75 , between 0.75 and 0.90 and $>0.90$ indicate poor, moderate, good, and excellent agreement, respectively. ${ }^{20}$ The vessel diameter measurements made by the two graders were averaged for further analysis. Bland-Altman plots were used for visual representation of the $95 \%$ limit of agreement between different types of scans, and paired t-test was used for quantitative comparison. A p value $<0.05$ was considered significant. The statistical software, Stata V.15, was used for statistical analyses.

\section{RESULTS}

A total of 27 eyes from 15 patients were included in the study of agreement of vessel diameter measured from two OCTA scan protocols $3 \times 3 \mathrm{~mm}^{2}$ and $9 \times 9 \mathrm{~mm}^{2}$ with AOO. Table 1 shows the demographics and clinical characteristics of these participants where the mean (SD) age was 30.87 (6.19) years, 67\% women and $80 \%$ Chinese. Their spherical equivalent (SD) was -3.82 (2.79). The OCTA's signal strength index (SD) of $3 \times 3 \mathrm{~mm}^{2}$ scans was 9.89 out of $10(0.33)$, while that of the $9 \times 9 \mathrm{~mm}^{2}$ scans was $9.87(0.35)$. The inter-rater agreement level was excellent for vessel diameter measured from either the OCTA protocols (ICC $=0.92$ for both $3 \times 3 \mathrm{~mm}^{2}$ and $9 \times 9 \mathrm{~mm}^{2}$ scan) or the AOO scans $(\mathrm{ICC}=0.98)$.

Table 2 summarises the vessel diameter measurement results obtained from the OCTA $3 \times 3 \mathrm{~mm}^{2}$, OCTA $9 \times 9 \mathrm{~mm}^{2}$ and AOO scans. The mean vessel diameter was widest measured from the OCTA $3 \times 3 \mathrm{~mm}^{2}(55.2 \pm 16.3 \mu \mathrm{m})$, followed by OCTA $9 \times 9 \mathrm{~mm}^{2}(54.7 \pm 14.3 \mu \mathrm{m})$ and narrowest by the AOO

Table 1 Clinical and ocular characteristics of participants $(n=15)$

\begin{tabular}{lc}
\hline Characteristics & Mean (SD), $\mathbf{n}(\%)$ \\
\hline Clinical characteristics & \\
\hline Age (yrs) & $30.87(6.19)$ \\
\hline Sex, male & 5 to 33.33 \\
\hline Ethnicity, Chinese & 12 to 80.00 \\
\hline Ocular characteristics & \\
\hline Imaged eye & 27 \\
\hline Right & 14 to 51.85 \\
\hline Left & 13 to 48.15 \\
\hline Spherical equivalent & $-3.82(2.79)$ \\
\hline
\end{tabular}

Table 2 Vessel diameter measurements in two OCTA protocols and AOO

\begin{tabular}{|c|c|c|c|}
\hline & A00 & $\begin{array}{l}\text { SS-OCTA } 3 \times 3 \mathrm{~mm}^{2} \\
\text { (OCTA3) }\end{array}$ & $\begin{array}{l}\text { SS-OCTA } 9 \times 9 \mathrm{~mm}^{2} \\
\text { (OCTA9) }\end{array}$ \\
\hline Unit: $\mu \mathrm{m}$ & Mean \pm SD (COV) & Mean \pm SD $(C O V)$ & Mean \pm SD $(C O V)$ \\
\hline Total $(n=240)$ & $50.5 \pm 15.6(30.9)$ & $55.2 \pm 16.3(29.5)$ & $54.7 \pm 14.3(26.1)$ \\
\hline Arteries $(n=147)$ & $51.8 \pm 13.9(26.8)$ & $56.7 \pm 14.5(25.6)$ & $56.1 \pm 13.2(23.5)$ \\
\hline Veins $(n=93)$ & $48.4 \pm 18.0(37.2)$ & $52.8 \pm 18.6(35.2)$ & $52.6 \pm 15.9(30.2)$ \\
\hline$D \geq 45 \mu m(n=133)$ & $60.8 \pm 13.5(22.1)$ & $65.4 \pm 14.4(22.0)$ & $63.5 \pm 13(20.5)$ \\
\hline$D<45 \mu \mathrm{m}(\mathrm{n}=107)$ & $37.7 \pm 5.1(13.7)$ & $42.4 \pm 6.7(15.8)$ & $43.7 \pm 5.7(13.1)$ \\
\hline
\end{tabular}

A00, adaptive optics ophthalmoscope; COV, coefficient of variance; OCTA, optical coherence tomography angiography; SS-OCTA, swept-source OCTA.

$(50.5 \pm 15.6 \mu \mathrm{m})$. The mean vessel diameter derived from either the OCTA $3 \times 3 \mathrm{~mm}^{2}$ or $9 \times 9 \mathrm{~mm}^{2}$ scans were significantly wider than AOO $(p<0.001)$. There were no differences in terms of the vessel diameter measurements between the two OCTA scans $(\mathrm{p}=0.298)$.

Table 3 summarises the differences in vessel diameter measurements between the different scans as categorised by vessel type and size. On average, the measurements obtained from both the OCTA scans were consistently $\sim 5 \mu \mathrm{m}$ wider than the AOO measurements regardless of vessel type (arterioles or venules). No significant difference was seen between the OCTA scan protocols $(p>0.05)$. When we examine vessels that were larger than $\geq 45 \mu \mathrm{m}$ (AOO: $60.8 \pm 13.5 \mu \mathrm{m}$ ), vessels measured from OCTA $3 \times 3 \mathrm{~mm}^{2}$ scan were significantly wider than that measured from the OCTA $9 \times 9 \mathrm{~mm}^{2}$ scan $(65.4 \pm 14.4 \mu \mathrm{m}$ vs $63.5 \pm 13 \mu \mathrm{m} ; \mathrm{p}=0.005)$. However, this effect was reversed for the smaller vessels (AOO: $37.7 \pm 5.1 \mu \mathrm{m}$ ), where vessels measured from the OCTA $3 \times 3 \mathrm{~mm}^{2}$ scan were marginally narrower than those from the OCTA $9 \times 9 \mathrm{~mm}^{2}$ scan $(42.4 \pm 6.7 \mu \mathrm{m}$ vs $43.7 \pm 5.7 \mu \mathrm{m} ; \mathrm{p}=0.009)$. Figure $2 \mathrm{~A}-\mathrm{C}$ shows the Bland-Altman plots, comparing the vessel diameter differences measured by different OCTA protocols and AOO.

\section{DISCUSSION}

We investigated OCTA-derived vessel diameter measurements by comparing with results from AOO. Our data showed that the mean vessel diameters measured from OCTA were significantly wider than AOO, regardless of scan protocols and vessel type. This finding may seem counterintuitive. One would expect that the vessels in the OCTA scans to appear narrower than the AOO scans because the blood cells proximal to the vessel wall tend to move at a much slower velocity, resulting in the absence of OCTA signals near the vessel wall. Instead, the vessel diameter appears wider when imaged with the OCTA system. There are three possible explanations. First, as explained by Spaide et al, ${ }^{21}$ OCTA system has a poorer lateral resolution compared with the AOO system, and also exhibits a low saturation threshold for the flow rate. Even when the laser beam spot partially hits on the vessel, the OCTA system can already detect red blood cell moving, resulting in an OCTA pixel above the noise floor that might contribute to the vessel diameter measurement. Second, the oversampling rate of the OCTA system may also play a role in the overestimation of the vessel width. In principle, the denser the sampling is, the more pixels a vessel contributes to the OCTA signal. This is clearly seen for large vessel imaging $(\mathrm{D} \geq 45 \mu \mathrm{m})$, where the OCTA $9 \times 9 \mathrm{~mm}^{2}$ tended to overestimate the vessel diameter marginally by $2.7 \mu \mathrm{m}$, whereas the overestimation is greater $(4.6 \mu \mathrm{m})$ when using the OCTA $3 \times 3 \mathrm{~mm}^{2}$. The OCTA $3 \times 3 \mathrm{~mm}^{2}$ protocol almost doubles the oversampling rate 
Table 3 Comparison of mean difference in vessel diameter $(\mu \mathrm{m})$ measured from two OCTA protocols and AOO

\begin{tabular}{|c|c|c|c|c|c|}
\hline Unit: $\mu \mathrm{m}$ & $\begin{array}{l}\text { Total } \\
(n=240)\end{array}$ & $\begin{array}{l}\text { Arteries } \\
(n=147)\end{array}$ & $\begin{array}{l}\text { Veins } \\
(n=93)\end{array}$ & $\begin{array}{l}D \geq 45 \mu \mathrm{m} \\
(\mathrm{n}=133)\end{array}$ & $\begin{array}{l}D<45 \mu \mathrm{m} \\
(\mathrm{n}=107)\end{array}$ \\
\hline Mean difference & 4.7 (4.0 to 5.4$)$ & 4.9 (3.9 to 5.8$)$ & 4.4 (3.3 to 5.5$)$ & 4.6 (3.5 to 5.7$)$ & 4.7 (3.8 to 5.7$)$ \\
\hline $\begin{array}{l}\text { OCTA3-AOO } \\
(95 \% \mathrm{Cl}) \\
\text { p value }\end{array}$ & $<0.001$ & $<0.001$ & $<0.001$ & 0.001 & $<0.001$ \\
\hline ICC (95\% Cl) & 0.94 (0.92 to 0.95$)$ & 0.91 (0.88 to 0.94$)$ & 0.96 (0.94 to 0.97$)$ & 0.90 (0.86 to 0.92 ) & 0.68 (0.57 to 0.77$)$ \\
\hline Mean difference & 4.2 (3.5 to 4.9$)$ & $4.3(3.4$ to 5.1$)$ & 4.1 (3.0 to 5.3 ) & 2.7 (1.7 to 3.7$)$ & 6.1 (5.3 to 6.9$)$ \\
\hline $\begin{array}{l}\text { OCTA9-A00 } \\
\text { (95\% CI) } \\
\text { p value }\end{array}$ & $<0.001$ & $<0.001$ & $<0.001$ & $<0.001$ & $<0.001$ \\
\hline ICC (95\% CI) & 0.93 (0.92 to 0.95$)$ & 0.92 (0.89 to 0.94$)$ & 0.95 (0.92 to 0.96$)$ & 0.90 (0.87 to 0.93 ) & 0.70 (0.59 to 0.79$)$ \\
\hline Mean difference & $-0.5(-1.3$ to 0.4$)$ & $-0.6(-1.8$ to 0.5$)$ & $-0.2(-1.6$ to 1.1$)$ & $-1.9(-3.2$ to -0.6$)$ & 1.3 (0.3 to 2.3$)$ \\
\hline $\begin{array}{l}\text { ОСТА9-0СТАЗ } \\
\text { (95\% CI) } \\
\text { p value }\end{array}$ & 0.298 & 0.301 & 0.729 & 0.005 & 0.009 \\
\hline ICC $(95 \% \mathrm{Cl})$ & 0.90 (0.87 to 0.92 ) & 0.87 (0.82 to 0.90$)$ & 0.93 (0.89 to 0.95 ) & 0.84 (0.78 to 0.88 ) & 0.66 (0.54 to 0.76 ) \\
\hline
\end{tabular}

$\mathrm{p}<0.05$ for values in bold

AOO, adaptive optics ophthalmoscope; ICC, intraclass correlation coefficient; OCTA, optical coherence tomography angiography.

compared with the $9 \times 9 \mathrm{~mm}^{2}$ protocol. Last but not the least is the effect of binarisation of OCTA images. We graded the vessel width from the binarised OCTA images, whereas we used the raw AOO image for grading. Because the OCTA pixel intensity has gradient, global thresholding will affect the number of pixels masked to be vessels. When the vessel contrast in OCTA is low, the binarisation effect may even counteract the broadening effect of OCTA. Nevertheless, further investigation is needed to fully understand the effect of binarisation on the vessel diameter measurement. Generally speaking, our finding confirms previous reports by other groups ${ }^{112122}$ that the vessel imaged using an OCTA tended to be larger than fundus photographs, and now the AOO scans.

Another factor that may affect the vessel diameter measurement using OCTA is the presence of the cell free plasma (CFP) layer. Previous studies using microscopy have shown that the width of the CFP layer is strongly correlated to the vessel diameter. ${ }^{23}$ The relative proportion decreases, however, from approximately $8 \%$ in vessels with a diameter of $10 \mu \mathrm{m}$ to $<6 \%$ in vessels of with a diameter of $50 \mu \mathrm{m} .{ }^{24}$ One would therefore assume that in the present study the relative error in OCTA measurements would be larger in the vessels with a diameter $\mathrm{D}<45 \mu \mathrm{m}$.

Ghasemi Falavarjani et al ${ }^{11}$ reported that the mean difference in the vessel calibre was $17.6 \mu \mathrm{m}$ between OCTA images and colour fundus images. We would like to stress that the differences may not be as great. The vessel diameter measured from OCTA images were approximately $4.7 \mu \mathrm{m}$ thicker than the AO measurements and this difference was marginally greater when using the OCTA $9 \times 9 \mathrm{~mm}^{2}$ on smaller vessels $(6.1 \mu \mathrm{m})$. The discrepancy might be attributed to the difference in specifications of the OCTA instruments and methods used for vessel diameter measurement in OCTA. On the other hand, Mo et $a^{25}$ compared the vessel diameter measurements from OCTA and AOSLO and found the difference to be around $5.7 \mu \mathrm{m}$, similar to our findings.

It was known that various factors such as the blood velocity, scan density, number of B-scan repetition and scan speed, may affect the signal-to-noise ratio (SNR) of OCTA images, which then influence the outcome of the quantitative OCTA analysis. In a wide variety of studies, retinal vasculature metrics, such as vessel density and perfusion density, were derived from binarised OCTA images and quantitatively analysed for diagnosis as well as prognosis performances. ${ }^{1426-29}$ Some of the metrics were calculated from skeletonised OCTA maps where vessels were shrunk down to lines with single-pixel width, while others incorporated the pixel area covered by the vessels in OCTA images. In the current study, we have used a prototype OCTA system which does not have built-in software for morphometry. Our goal was to answer a fundamental question, which
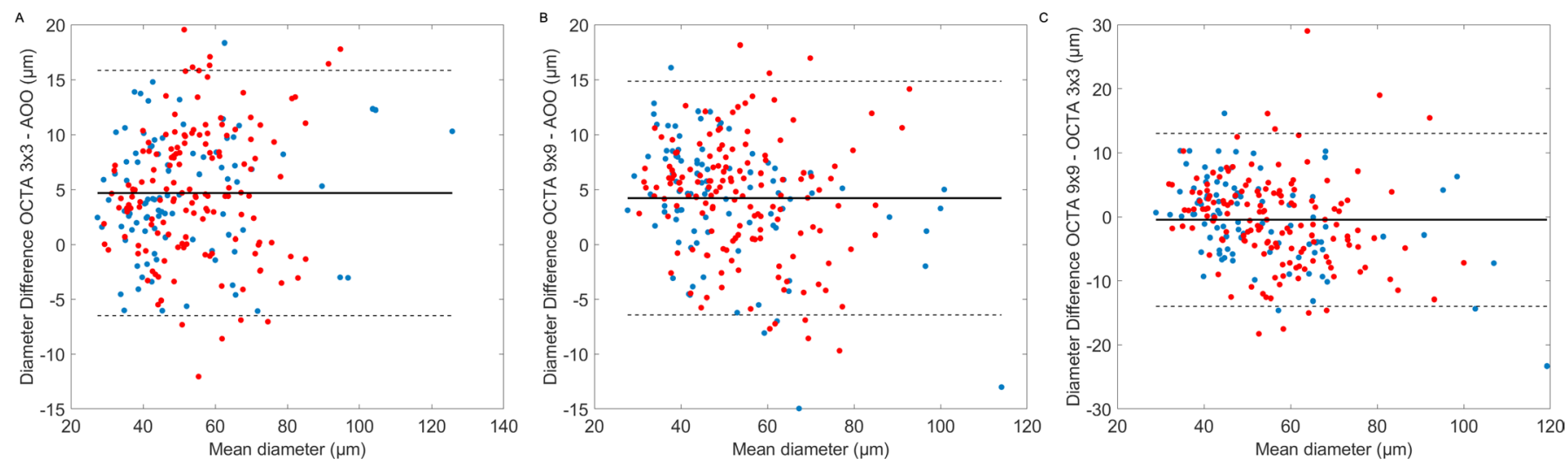

Figure 2 Bland-Altman plots of vessel diameter measurements from OCTA $9 \times 9 \mathrm{~mm}^{2}, 3 \times 3 \mathrm{~mm}^{2}$ and AOO scans. Red dots: arterioles. Blue dots: venules. A00, adaptive optics ophthalmoscope; OCTA, optical coherence tomography angiography. 
is related to the reliability of the commonly used OCTA vessel density measurement when using different scan protocols. Since different vendors use different algorithms for calculating vessel density this may have an effect on the absolute numbers of vessel density as well. Some investigators found significant differences in OCTA metrics between different machines and different algorithms, ${ }^{30-32}$ while others did not. ${ }^{33}$ We found the scan density of OCTA protocol may affect the accuracy of vessel diameter measurement and therefore influence the calculation of OCTA metrics. To which degree this effect may also add to the poor comparability of data with different instruments ${ }^{34}$ remains to be investigated. On the contrary, metrics, for example, vessel density, derived from skeletonizsed OCTA images may be less affected by different OCTA protocols or instruments, as suggested by other studies as well. ${ }^{2627}$

Conventionally, retinal vessel calibres are extracted from digitalised colour fundus photographs using computer-assisted programmes, and yet different image processing methods may lead to different vessel diameter measurement results. ${ }^{12} 13$ Moreover, the vessel width as extracted from colour fundus photograph may only partially reflect the actual vessel lumen. ${ }^{35}$ Previous researchers tried to use the OCT to measure vessel calibre in the axial direction (vertically) that is insensitive to the ocular magnification effect. ${ }^{12}{ }^{36}$ However, the accuracy of this approach is dependent on the coherence length of the light source, which is usually above $5 \mu \mathrm{m}$ in tissue. The transverse resolution of AOO $(2 \mu \mathrm{m})$ is much better than that of conventional fundus photography as well as OCTA or OCT systems. Even though the AOO system does not correct for ocular magnification effect, the vessel diameter measurements from the AOO images would still be closer to the ground truth. In addition, the acquisition time of AOO is very short (2s), making it almost impervious to motion artefact. Therefore, using $\mathrm{AOO}$ as a reference is more reasonable and most likely induces no relevant error to our conclusions. It should also be noted that the AOO vessel diameter measurement is heavily dependent on proper focusing. To limit the influence of focusing errors the images included in the study passed quality control.

There are also some limitations of this study. The sample size was relatively small, and only healthy subjects were included. As discussed above, the vessel diameter measurement was conducted on binarised OCTA images. Future studies may consider the effect of varying the thresholding method on the vessel diameter measurements. Both AOO and OCTA measurements did not count for the magnification error of the eye, and therefore may still deviate from the vessel diameter in actuality. We only included one SS-OCTA instrument in this study and the result may not be generalised to other OCTA devices. The vessels analysed in our study were mostly arterioles/venules and the results might not be generalised to the smaller vessels such as capillaries, which consists of a major portion during the calculation of vessel density and perfusion density.

In conclusion, we found that vessels measured from OCTA appeared to be thicker than those in AOO and different OCTA protocols may affect the vessel diameter measurement. This needs to be taken into consideration when vessel diameters or metrics, such as vessel density and perfusion density that implicitly incorporate vessel diameters, are extracted from OCTA images.

Twitter Xinwen Yao @XinwenYao

Contributors $X Y, L S$ and JC conceived the concept and designed the study. EL collected the data. XY, MK and YH performed the image processing and data analysis. XY drafted the manuscript. DWKW and BT offered administrative or technical support. LS and JC coordinated the project and handled the funding. All contributed to the final manuscript.

Funding The study is funded by National Medical Research Council (grants CG/ C010A/2017, OFLCG/004C/2018 and TA/MOH-000249-00/2018) and the Duke-NUS Medical School (Duke-NUS-KP(Coll)/2018/0009A), Singapore.

Disclaimer The sponsor or funding organisation had no role in the design or conduct of this research. The funders had no role in the design and conduct of the study; collection, management, analysis and interpretation of the data; and preparation, review or approval of the manuscript; and decision to submit the manuscript for publication.

Competing interests None declared.

Patient consent for publication Not required.

Provenance and peer review Not commissioned; externally peer reviewed.

Data availability statement Data are available upon reasonable request. All raw data and measurement results are deidentified participant data, and are available upon reasonable request. Please contact Dr Jacqueline Chua: jacqueline.chua.y.m@ seri.com.sg if interested.

Open access This is an open access article distributed in accordance with the Creative Commons Attribution Non Commercial (CC BY-NC 4.0) license, which permits others to distribute, remix, adapt, build upon this work non-commercially, and license their derivative works on different terms, provided the original work is properly cited, appropriate credit is given, any changes made indicated, and the use is non-commercial. See: http://creativecommons.org/licenses/by-nc/4.0/.

\section{ORCID iDs}

Xinwen Yao http://orcid.org/0000-0001-8813-4676

Leopold Schmetterer http://orcid.org/0000-0002-7189-1707

Jacqueline Chua http://orcid.org/0000-0002-6474-5293

\section{REFERENCES}

1 Kashani AH, Chen C-L, Gahm JK, et al. Optical coherence tomography angiography: a comprehensive review of current methods and clinical applications. Prog Retin Eye Res 2017:60:66-100.

2 Chua J, Tan B, Ang M, et al. Future clinical applicability of optical coherence tomography angiography. Clin Exp Optom 2019;102:260-9.

3 Ang M, Tan ACS, Cheung CMG, et al. Optical coherence tomography angiography: a review of current and future clinical applications. Graefes Arch Clin Exp Ophthalmol 2018;256:237-45

4 Hong J, Tan B, Quang ND, et al. Intra-session repeatability of quantitative metrics using widefield optical coherence tomography angiography (OCTA) in elderly subjects. Acta Ophthalmol 2019

5 Ikram MK, Ong YT, Cheung CY, et al. Retinal vascular caliber measurements: clinical significance, current knowledge and future perspectives. Ophthalmologica 2013;229:125-36.

6 Sun C, Wang JJ, Mackey DA, et al. Retinal vascular caliber: systemic, environmental, and genetic associations. Surv Ophthalmol 2009;54:74-95.

7 Klein R, Klein BEK, Moss SE, et al. The relation of retinal vessel caliber to the incidence and progression of diabetic retinopathy: XIX: the Wisconsin epidemiologic study of diabetic retinopathy. Arch Ophthalmol 2004;122:76-83.

8 Jonas JB, Wang N, Wang S, et al. Retinal vessel diameter and estimated cerebrospinal fluid pressure in arterial hypertension: the Beijing eye study. Am J Hypertens 2014;27:1170-8

9 Klein R, Knudtson MD, Klein BEK, et al. The relationship of retinal vessel diameter to changes in diabetic nephropathy structural variables in patients with type 1 diabetes. Diabetologia 2010;53:1638-46.

10 Heitmar R, Lip GYH, Ryder RE, et al. Retinal vessel diameters and reactivity in diabetes mellitus and/or cardiovascular disease. Cardiovasc Diabetol 2017;16:56.

11 Ghasemi Falavariani K, Al-Sheikh M, Darvizeh F, et al. Retinal vessel calibre measurements by optical coherence tomography angiography. Br J Ophthalmol 2017;101:989-92.

12 Fondi K, Aschinger GC, Bata AM, et al. Measurement of retinal vascular caliber from optical coherence tomography phase images. Invest Ophthalmol Vis Sci 2016;57:0CT121-9.

13 Ouyang Y, Shao Q, Scharf D, et al. Retinal vessel diameter measurements by spectral domain optical coherence tomography. Graefes Arch Clin Exp Ophthalmol 2015;253:499-509.

14 Paques M, Meimon S, Rossant F, et al. Adaptive optics ophthalmoscopy: application to age-related macular degeneration and vascular diseases. Prog Retin Eye Res 2018;66:1-16

15 Koch E, Rosenbaum D, Brolly A, et al. Morphometric analysis of small arteries in the human retina using adaptive optics imaging: relationship with blood pressure and focal vascular changes. J Hypertens 2014;32:890-8 
16 Chua J, Chee ML, Chin CWL, et al. Inter-relationship between ageing, body mass index, diabetes, systemic blood pressure and intraocular pressure in Asians: 6-year longitudinal study. Br J Ophthalmol 2019;103:196-202.

17 Chua J, Chin CWL, Hong J, et al. Impact of hypertension on retinal capillary microvasculature using optical coherence tomographic angiography. J Hypertens 2019;37:572-80.

18 Chua J, Chin CWL, Tan B, et al. Impact of systemic vascular risk factors on the choriocapillaris using optical coherence tomography angiography in patients with systemic hypertension. Sci Rep 2019;9:5819.

19 Otsu N. A threshold selection method from Gray-Level histograms. IEEE Trans Syst Man Cybern 1979;9:62-6.

20 Koo TK, Li MY. A guideline of selecting and reporting intraclass correlation coefficients for reliability research. J Chiropr Med 2016;15:155-63.

21 Spaide RF, Fujimoto JG, Waheed NK. Image artifacts in optical coherence tomography angiography. Retina 2015;35:2163-80.

22 Salas M, Augustin M, Ginner L, et al. Visualization of micro-capillaries using optical coherence tomography angiography with and without adaptive optics. Biomed Opt Express 2017;8:207-22.

23 Kim S, Kong RL, Popel AS, et al. A computer-based method for determination of the cell-free layer width in microcirculation. Microcirculation 2006;13:199-207.

24 Kim S, Kong RL, Popel AS, et al. Temporal and spatial variations of cell-free layer width in arterioles. Am J Physiol Heart Circ Physiol 2007;293:H1526-35.

25 Mo S, Krawitz B, Efstathiadis E, et al. Imaging foveal microvasculature: optical coherence tomography angiography versus adaptive optics scanning light ophthalmoscope fluorescein angiography. Invest Ophthalmol Vis Sci 2016;57:0CT130-40.

26 Hirano T, Kitahara J, Toriyama Y, et al. Quantifying vascular density and morphology using different swept-source optical coherence tomography angiographic scan patterns in diabetic retinopathy. Br J Ophthalmol 2019;103:216-21.
27 Jia Y, Bailey ST, Hwang TS, et al. Quantitative optical coherence tomography angiography of vascular abnormalities in the living human eye. Proc Natl Acad Sci U S A 2015;112:E2395-402.

28 Jia Y, Wei E, Wang $X$, et al. Optical coherence tomography angiography of optic disc perfusion in glaucoma. Ophthalmology 2014;121:1322-32.

29 Tey KY, Teo K, Tan ACS, et al. Optical coherence tomography angiography in diabetic retinopathy: a review of current applications. Eye Vis 2019;6:37.

30 Corvi F, Pellegrini M, Erba S, et al. Reproducibility of vessel density, fractal dimension, and foveal avascular zone using 7 different optical coherence tomography angiography devices. Am J Ophthalmol 2018;186:25-31.

31 Lu Y, Wang JC, Zeng R, et al. Quantitative comparison of microvascular metrics on three optical coherence tomography angiography devices in chorioretinal disease. Clin Ophthalmol 2019;13:2063-9.

32 Rabiolo A, Gelormini F, Sacconi R, et al. Comparison of methods to quantify macular and peripapillary vessel density in optical coherence tomography angiography. PLOS One 2018;13:e0205773.

33 Munk MR, Giannakaki-Zimmermann H, Berger L, et al. OCT-angiography: a qualitative and quantitative comparison of 4 OCT-A devices. PLoS One 2017;12:e0177059.

34 Corvi F, Cozzi M, Barbolini E, et al. Comparison between several optical coherence tomography angiography devices and indocyanine green angiography of choroidal neovascularization. Retina 2020;40:873-80.

35 International Society for Optics and Photonics. Automatic determination of the artery-vein ratio in retinal images. Medical imaging 2010: computer-aided diagnosis, 2010.

36 Hosseinaee Z, Tan B, Martinez A, et al. Comparative study of optical coherence tomography angiography and Phase-Resolved Doppler optical coherence tomography for measurement of retinal blood vessels caliber. Trans/ Vis Sci Technol 2018;7:18. 